\title{
Modal Analysis of Vertical Wind Turbine's Frame and Impeller
}

\author{
${ }^{1}$ Rajivranjan Tiwari, ${ }^{2}$ Aseem Chandra Tiwari
}

\begin{abstract}
Increasing demand in energy facilitated the need of clean energy such as wind energy. In thisproject modal analysis has been used to determine the natural frequencies and the mode shapesof frame and impeller of vertical axis wind turbine with impeller. FEA consists of a computer model of a material. Analyzed for specific results. FEA uses a complex system of points called nodes which make a grid called a mesh. Nodes are assigned at a certain density throughout the material depending on the anticipated stress levels of a particular area. Regions which will receive large amounts of stress usually have a higher node density than those which experience little or no stress. Points of interest may consist of: fracture point of previously tested material, fillets, corners, complex detail, and high stress areas. The mesh acts like a spider web in that from each node, there extends a mesh element to each of the adjacent nodes. This web of vectors is what carries the material properties to the object, creating many elements. The natural frequencies originating from the FE-modelingand the modal analysis, respectively, are observed. Comparing the mode shapesgood agreement has been demonstrated for the dominating deflectiondirection. For the non-dominating deflection directions, the qualitative featuresof measured and computed modes shapes are in good agreement.
\end{abstract}

Key words: Free vibration, Finite Element Method, Frame, Impeller.

\section{Introduction}

The frame is the basic component to support the essential parts of the wind turbine. It must be sturdy enough to compensate the static forces such as the weight of the wind turbine and tightening forces due to screws or bolts, and particularly dynamic forces such as vibrations due to rotating elements and fluctuating thrust force over the turbine due to blowing wind.

Impellers are components are used to push the air through air ducts to condition the air of buildings. Their efficiency is generally about $65 \%$ for blowing purposes; however this is not known when they are used for converting wind energy into electricity. They will be used in this thesis due to their strong structure and ease of assembly. Due to their forward curved shape, they are working with both drag and lift forces and to improve their efficiency as a wind turbine, they are recommended to be used with guide vanes.

\section{Procedure For Analysis}

\section{Preprocessing:}

- CAD Modeling : Creation of CAD Model by using the CAD modeling tools UG for creating the geometry of the part/assembly of which we want to perform FEA.

- Importing 3D CAD geometry to FEA Package: Start the FEA package and import the CAD geometry into the FEA package.

- Defining Material Properties: We need to tell the FEA package which material we are using for the part. By this process we have to tell modulus of elasticity, Poisson's ratio and all other necessary properties require for the FEA.

- Meshing: Meshing is a critical operation in FEA. In this operation, the CAD geometry is divided into large numbers of small pieces. The small pieces are called mesh. The analysis accuracy and duration depends on the mesh size and orientations. With the increase in mesh size, the finite element analysis speed increase but the accuracy decrease.

- Defining Boundary Condition: we have to tell the FEA package where we want to apply loads and where we want to rest the part. (constraints).

\section{Solution or Analysis}

- $\quad$ Solve: In this step you tell the FEA package to solve the problem for the defined material properties, boundary conditions and mesh size.

\section{Post processing.}

- Post Processing: For viewing and interpretation of Result. The result can be viewed in various formats: graph, value, animation etc. 
2.1.1 Cad Model of Frame And Impeller:-
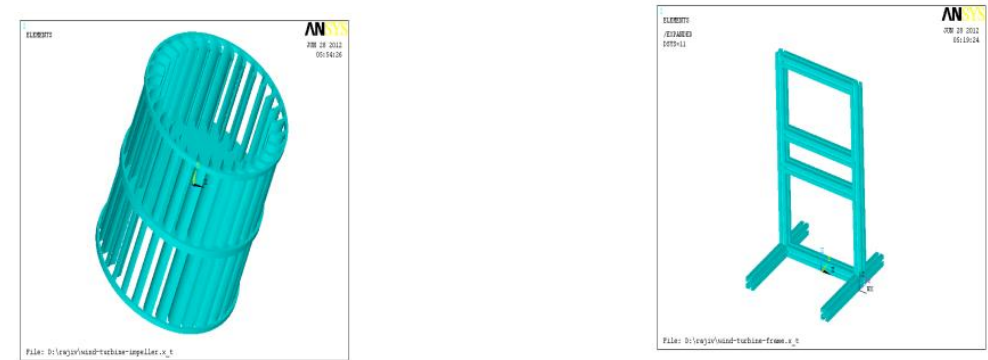

2.1.2 Material Properties Of Frame And Impeller Young Modulus ofAluminum Frame -70 X $10^{3} \mathrm{Mpa}$

Poisson Ratio- 0.33

Ultimate Tensile Strength $-2790 \mathrm{~kg} / \mathrm{mm} 3$

\subsubsection{MESHING OF FRAMES:-}

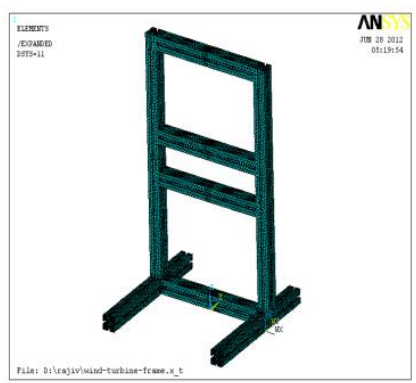

Element type- solid 187 (10node 187) Tetrahedral is taken.

Element size-10

No. OF Nodes -131528

No. Of Element - 68482

\subsubsection{Meshing Of Impellers:-}

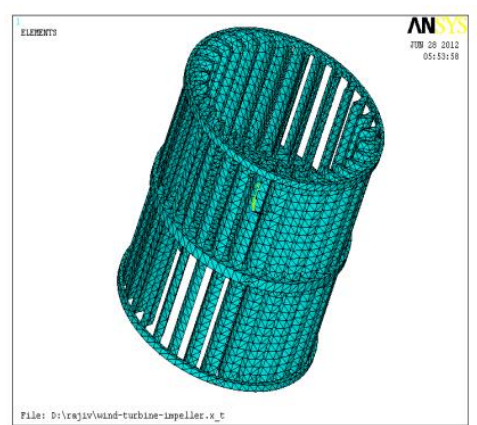

Element type- solid 187 (10node 187) Tetrahedral is taken.

Element size-10

No. OF Nodes -57128

No. Of Element - 25459

\subsubsection{Boundary Conditions :-}

Frame :- It is fixed on a base frame. No load is applied.

Impeller :- It is mounted at center shaft of frame( not shown here. It is separate part itself) It moves with wind velocity freely.

\subsubsection{Analysis Of Frame:-}

Type of Analysis :- Modal ( free vibration) analysis.

No. of modes : 5 

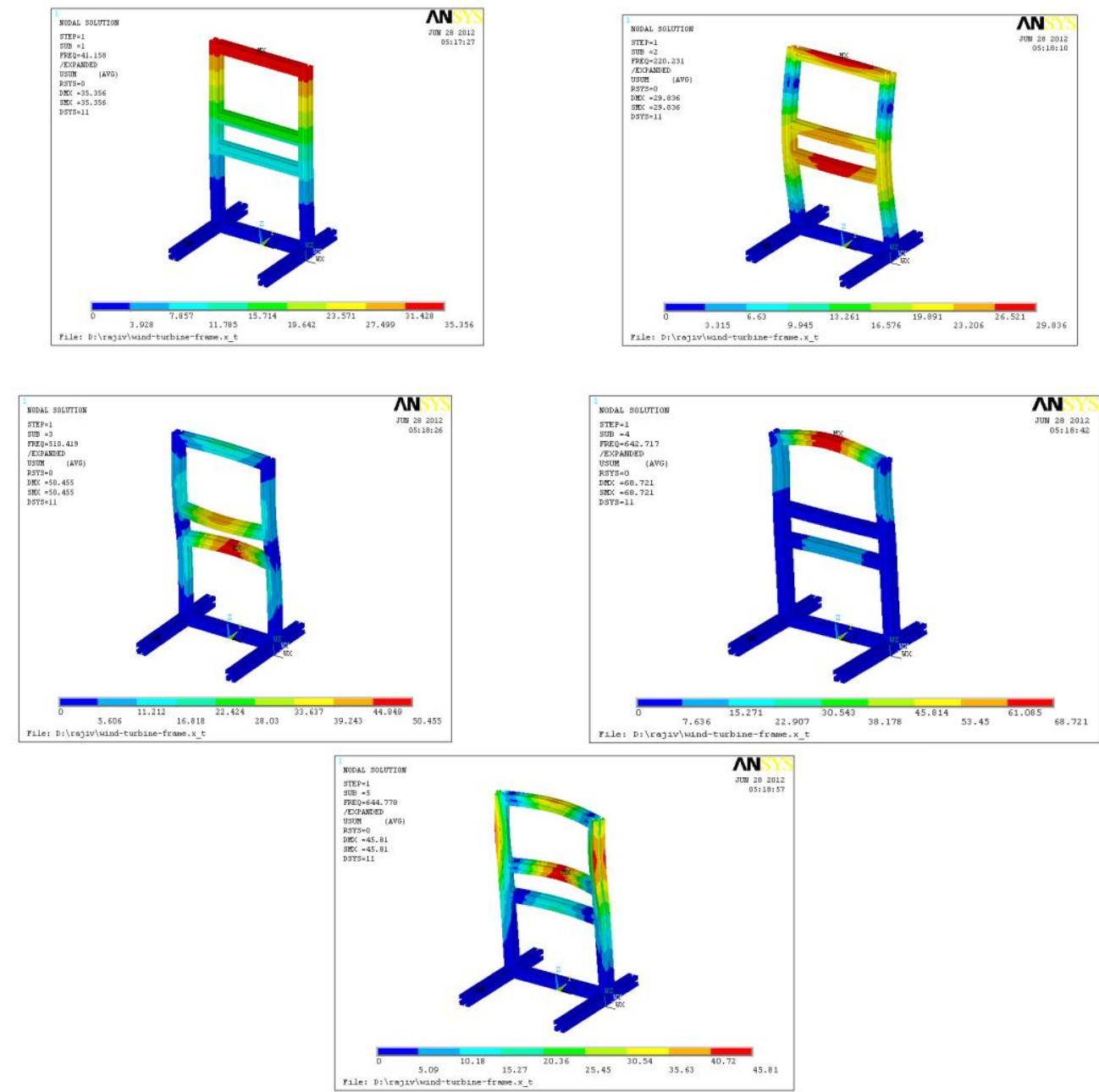

2.2.2 Analysis Of Impeller

Type of Analysis :- Modal ( free vibration) analysis.

No. of modes : 5
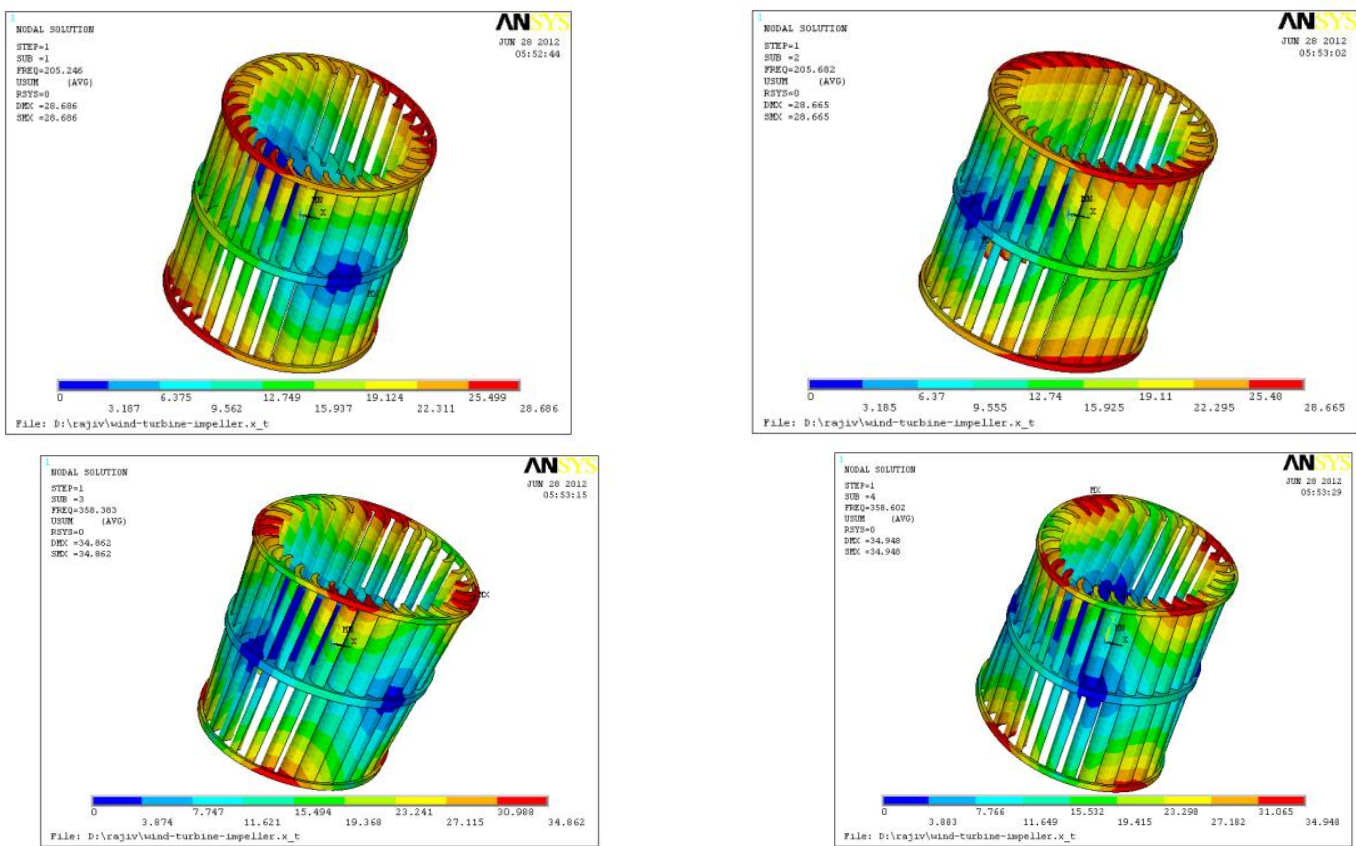


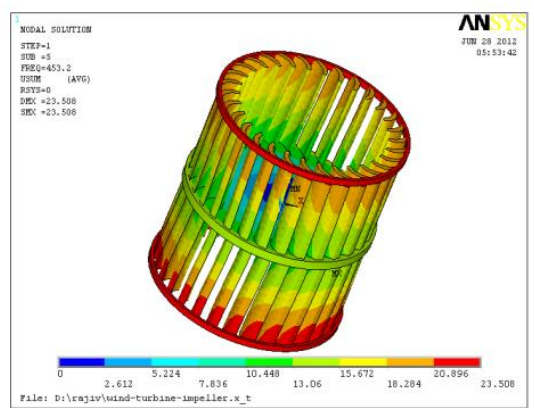

3.1 Tabular Chart Of Frame :-

\section{Result And Discussion}

\begin{tabular}{|c|c|c|c|c|c|c|c|c|c|}
\hline $\begin{array}{c}\text { APPLIED } \\
\text { FREQUENCY }\end{array}$ & \multicolumn{10}{|c|}{$\begin{array}{c}\text { DEFLECTION RATE } \\
(\text { Hertz) }\end{array}$} & $\mathrm{X} 1$ & $\mathrm{X} 2$ & $\mathrm{X} 3$ & $\mathrm{X} 4$ & $\mathrm{X} 5$ & $\mathrm{X} 6$ & $\mathrm{X} 7$ & $\mathrm{X} 8$ & $\mathrm{X} 9$ \\
\cline { 2 - 11 } & & & & & & & \\
\hline 41.158 & 3.928 & 7.857 & 11.785 & 15.714 & 19.642 & 23.571 & 27.499 & 31.428 & 35.356 \\
\hline 220.231 & 3.315 & 6.639 & 9.945 & 13.361 & 16.576 & 19.891 & 23.206 & 26.521 & 29.836 \\
\hline 510.419 & 5.606 & 11.213 & 16.818 & 22.424 & 28.03 & 33.637 & 39.243 & 44.849 & 50.455 \\
\hline 642.717 & 7.636 & 15.271 & 22.907 & 30.543 & 38.178 & 45.814 & 53.45 & 61.085 & 68.721 \\
\hline 644.778 & 5.09 & 10.18 & 15.27 & 20.36 & 25.45 & 30.54 & 35.63 & 40.72 & 45.81 \\
\hline
\end{tabular}

3.2 Tabular Chart Of Impeller:-

\begin{tabular}{|c|c|c|c|c|c|c|c|c|c|}
\hline \multirow{2}{*}{$\begin{array}{c}\text { APPLIED } \\
\text { FREQUENCY } \\
\text { (Hertz) }\end{array}$} & \multicolumn{9}{|c|}{$\begin{array}{l}\text { DEFLECTION RATE } \\
(\mathrm{mm})\end{array}$} \\
\hline & $\mathrm{X} 1$ & $\mathrm{X} 2$ & X3 & $\mathrm{X} 4$ & X5 & X6 & X7 & X8 & X9 \\
\hline 205.246 & 3.187 & 6.375 & 9.562 & 12.749 & 15.937 & 19.124 & 22.311 & 25.499 & 28.686 \\
\hline 205.682 & 3.185 & 6.37 & 9.555 & 12.74 & 15.925 & 19.11 & 22.295 & 25.48 & 28.665 \\
\hline 358.383 & 3.874 & 7.747 & 11.621 & 15.494 & 19.368 & 23.241 & 27.115 & 30.988 & 34.862 \\
\hline 358.602 & 3.883 & 7.766 & 11.649 & 15.532 & 19.415 & 23.298 & 27.182 & 31.065 & 34.948 \\
\hline 453.2 & 2.612 & 5.224 & 7.836 & 10.448 & 13.06 & 15.672 & 18.284 & 20.896 & 23.508 \\
\hline
\end{tabular}

1. With increase in Vibration the deflection rate increases linearly..

2. For small increase in vibration the values of deflection rate does not necessarily increases but varies positively or negatively from previous reading.

3. In analysis diagram different mode shapes are shown for different frequencies for parts of frame and impeller

\section{Conclusion}

In this project modal analysis proves that it can be used to determine modal characteristics offrame and impeller. The most important properties like naturalfrequencies and the mode shapes can be determined experimentallyby the modal analysis method.The analysis show that the results are very similar, when the analysis are repeated. It is concluded that the method is very reliable, with a high ability to repeat it self.

\section{References}

[1]. Applied Modal Analysis of Wind Turbine Blades by HenrikBroen Pedersen, Ole Jesper Dahl KristensenRisø National Laboratory, Roskilde (Risø-R-1388(EN) DenmarkFebruary 2003

[2]. D. J. Ewins: Modal Testing: Theory, Practice and Application

[3]. Forward curved centrifugal TABLOCK Blower Wheels( for dimension of impeller)

[4]. kjnAluminiumProfile \& Accessories. [cited 2010 September 18]; Available from: http://www.aluminium-profile.co.uk.

[5]. C.F.Beards, Engineering Vibration Analysis with Application to Control Systems. 